Journal for ImmunoTherapy of Cancer

\title{
Early toxicity and clinical outcomes after chimeric antigen receptor $T$-cell (CAR-T) therapy for lymphoma
}

\begin{abstract}
Jonathan E Brammer, ${ }^{1}$ Zachary Braunstein (D) ,2 Aashish Katapadi, ${ }^{3}$ Kyle Porter, ${ }^{4}$ Michael Biersmith, ${ }^{3}$ Avirup Guha, ${ }^{3,5}$ Sumithira Vasu, ${ }^{1}$ Vedat O Yildiz, ${ }^{4}$ Sakima A Smith, ${ }^{3}$ Benjamin Buck, ${ }^{3}$ Devin Haddad, ${ }^{3}$ Richard Gumina, ${ }^{3}$ Basem M William, ${ }^{1}$ Sam Penza, ${ }^{1}$ Ayman Saad, ${ }^{1}$ Nathan Denlinger, ${ }^{6}$ Ajay Vallakati, ${ }^{3}$ Ragavendra Baliga, ${ }^{3}$ Raymond Benza, ${ }^{3}$ Philip Binkley, ${ }^{3}$ Lai Wei, ${ }^{4}$ Mason Mocarski, ${ }^{2}$ Steven M Devine, ${ }^{7}$ Samantha Jaglowski, ${ }^{1}$ Daniel Addison ${ }^{3,8}$
\end{abstract}

To cite: Brammer JE, Braunstein Z, Katapadi A, et al. Early toxicity and clinical outcomes after chimeric antigen receptor T-cell (CAR-T) therapy for lymphoma. Journal for ImmunoTherapy of Cancer 2021;9:e002303. doi:10.1136/ jitc-2020-002303

- Additional supplemental material is published online only. To view, please visit the journal online (http://dx.doi.org/10. 1136/jitc-2020-002303).

JEB and ZB contributed equally. Accepted 09 July 2021

Check for updates

(C) Author(s) (or their employer(s)) 2021. Re-use permitted under CC BY-NC. No commercial re-use. See rights and permissions. Published by BMJ.

For numbered affiliations see end of article.

Correspondence to

Daniel Addison;

daniel.addison@osumc.edu

\section{ABSTRACT}

Background Chimeric antigen receptor T-cell (CAR-T) infusion is associated with early toxicity. Yet, whether early toxicity development holds ramifications for long-term outcomes is unknown.

Methods From a large cohort of consecutive adult patients treated with CAR-T therapies for relapsed or refractory lymphomas from 2016 to 2019, we assessed progression-free survival (PFS), by toxicity development (cytokine release syndrome (CRS), neurotoxicity, or cardiotoxicity]. We also assessed the relationship of toxicity development to objective disease response, and overall survival (OS). Multivariable regression was utilized to evaluate relationships between standard clinical and laboratory measures and disease outcomes. Differences in outcomes, by toxicity status, were also assessed via 30-day landmark analysis. Furthermore, we assessed the effects of early anti-CRS toxicity therapy use (at $\leq$ grade 2 toxicity) on maximum toxicity grade observed, and longterm disease outcomes (PFS and OS).

Results Overall, from 102 CAR-T-treated patients, 90 were identified as treated with single-agent therapy, of which $88.9 \%$ developed toxicity ( 80 CRS, 41 neurotoxicity, and 17 cardiotoxicity), including $28.9 \%$ with high-grade $(\geq 3)$ events. The most common manifestations were hypotension at $96.6 \%$ and fever at $94.8 \%$. Among patients with cardiac events, there was a non-significant trend toward a higher prevalence of concurrent or preceding high-grade ( $\geq 3)$ CRS. $50.0 \%$ required tocilizumab or corticosteroids. The median time to toxicity was 3 days; high grade CRS development was associated with cardiac and neurotoxicity. In multivariable regression, accounting for disease severity and traditional predictors of disease response, moderate (maximum grade 2) CRS development was associated with higher complete response at 1 year (HR: 2.34; $p=0.07$ ), and longer PFS (HR: $0.41 ; p=0.02$, in landmark analysis), and OS (HR: 0.43; $p=0.03$ ). Among those with CRS, relative blood pressure (HR: 2.25; $p=0.004$ ), respectively, also associated with improved PFS. There was no difference in disease outcomes, or maximum toxicity grade (CRS, neurotoxicity, or cardiotoxicity) observed, based on the presence or absence of the use of early CRS-directed therapies.
Conclusions Among adult lymphoma patients, moderate toxicity manifest as grade 2 CRS after CAR-T infusion may associate with favorable clinical outcomes. Further studies are needed to confirm these findings.

\section{INTRODUCTION}

Chimeric antigen receptor T-cell (CAR-T) therapies have rapidly transformed the treatment of advanced hematologic malignancies. ${ }^{1-7}$ Initially FDA approved in 2017 , anti-CD19 CAR-T therapies are associated with dramatically improved outcomes among patients with relapsed and refractory lymphoid malignancies, where prognosis was previously poor. ${ }^{1-8}$ Due to mounting evidence of preclinical and clinical efficacy suggesting benefit even beyond lymphoid malignancies, $>550$ additional clinical trials are currently ongoing. ${ }^{9}$

Despite CAR-T's benefits, a significant number of patients do not see sustained response, with only $40 \%$ seeing longerterm progression-free survival (PFS). ${ }^{1-5} 10$ Available data have also shown variation in objective disease responses, with 54\%-90\% of patients responding depending on the population studied, and to date, there are no known early predictors of response. ${ }^{1-5}$ This is compounded by the common nature of immune-related toxicities, including cytokine release syndrome (CRS), neurotoxicity, and cardiotoxicity. ${ }^{10-13}$ Yet, currently there is limited evidence to guide the prognostication of outcomes after treatment with CAR-T therapies.

Recently, a growing number of reports have suggested potential relationships between the development of early immune-related toxicities and long-term therapeutic efficacy after the initiation of several immunomodulatory 
therapies. ${ }^{14-19}$ This is particularly true of therapies with broad T-cell modulatory and/or autoimmunity-related effects. ${ }^{17}$ Among patients treated with approved CAR-T therapies, with T-cell engagement and subsequent broad immunomodulatory effects, over $80 \%$ experience at least one low-grade adverse event within days of infusion. ${ }^{1-5}$ Yet, whether the development of CAR-T-related toxicities has ramifications on longer term outcomes after treatment initiation remains unknown.

\section{METHODS}

From consecutive patients treated with CAR-T therapy for relapsed/refractory diffuse large B-cell lymphoma (DLBCL), follicular lymphoma, or mantle-cell lymphoma at The Ohio State University's Comprehensive Cancer Center from January 2016 through Decemeber 2019, we evaluated the profile and outcomes of incident CRS, after Institutional Review Board approval. We also assessed the incidence of non-CRS toxicities, including cardiotoxic and neurotoxic events. Study patients included adults $\geq 18$ years of age treated with CAR-T for a relapsed or refractory lymphomas. Patients with incomplete medical records for the cancer, cardiovascular, or neurologic variables of interest were excluded. Patients treated with clinical trial combinations of CAR-T and other immunomodulatory experimental therapies were excluded. Baseline characteristics, including medical history, and previous treatment were evaluated. Disease bulk was evaluated and bulky disease defined as having a primary mass that is $\geq 10 \mathrm{~cm}$, based on imaging (ie, positron emission tomography (PET) or CT). Incident (new) CRS was defined according to consensus criteria. ${ }^{20}$ Toxicities included those events meeting established Common Terminology Criteria for Adverse Events (CTCAE) within 30 days of infusion. ${ }^{21}$ We specifically considered cardiotoxicity given increasing evidence of potential profound or limiting events. ${ }^{12}{ }^{13}$ Baseline and preceding anticancer therapies were also recorded. Disease response was assessed per Lugano consensus criteria. ${ }^{22}$ Moreover, we manually searched all subject charts for incident adverse events, inclusive of CRS, neurotoxicity, and cardiotoxicity, in addition to cardiovascular or all-cause death.

\section{Toxicity definitions and grading}

Toxicity was considered as events within 30 days of CAR-T infusion or during index-admission. Individual CRS components were assessed according to consensus (Penn) criteria. $^{20}$ Specifically, fever, coagulopathy, hypoxia, hypotension, and end organ toxicity (eg, renal failure) were included as criteria for incident or worsening CRS (online supplemental table 1). Fever was defined as oral temperature $>38^{\circ} \mathrm{C}$, and hypoxia was considered oxygen saturation $<90 \%$ on room air or need for supplemental oxygen, while hypotension was considered as minimum systolic blood pressure (SBP) of $<105 \mathrm{~mm} \mathrm{Hg}$. We selected $<105 \mathrm{~mm} \mathrm{Hg}$ due to the desire to capture even those with probable relative hypotension. Neurotoxicity grades included encephalopathy, seizure, dysphasia, tremor, headache, confusion, depressed level of consciousness, and cerebral edema. ${ }^{20}$ Furthermore, CVD events were defined as myocarditis, congestive heart failure, stroke, myocardial infarction, symptomatic arrhythmia (eg, atrial fibrillation ventricular tachycardia), or cardiac death. ${ }^{12} 13$

\section{Clinical laboratory measures}

Available clinical laboratory measures, including c-reactive protein (CRP), ferritin, complete blood counts with differential, troponin, alanine aminotransferase, and lactate dehydrogenase (LDH) were also considered. These were widely available and routinely measured among treated patients, including those with concern for toxicity. ${ }^{23} 24$ The maximum value was included where multiple measures were obtained.

\section{Outcomes}

The primary outcome was the development of cancer disease progression or death (ie, PFS) after CAR-T initiation. The secondary outcome was the occurrence of partial or complete response at 1 year, and overall survival. Follow-up began from the time of CAR-T initiation. Toxicity events were graded using CTCAE V.5, and Lugano consensus criteria. Moreover, we assessed the incidence and outcomes CRS, by CAR-T product employed (axicabtagene ciloleucel (axi-cel), and tisagenlecleucel (tisa-cel), respectively). Axicabtagene ciloleucel and brexucabtagene autoleucel were considered together as axicel, as the two products are clinically identical, with some difference in T-cell enrichment/processing.

\section{Statistical analysis}

Descriptive statistics were used to summarize patient characteristics, using mean \pm SD or median (IQR) for continuous variables and frequency counts with percentages for categorical variables. Time-to-event analysis methods were used to summarize PFS and evaluate associations with these outcomes. Patients without progression or death were considered censored at the last follow-up date. PFS was estimated and displayed by Kaplan-Meier curves. Cox proportional hazards models were used to assess association of patient factors with PFS. Univariable models were fit using maximum CRS grade as the independent variable, followed by using individual CRS components, neurotoxicity, and patient laboratory values. Multivariable Cox proportional hazards models were then fit using potential risk factors identified in the univariable models. To avoid overfitting, we considered the number of PFS events when evaluating how many independent variables to include in the models. Log-rank tests and KaplanMeier curves were also used to compare PFS by product type received. Furthermore, $\chi^{2}$ tests were used to assess differences in CRS grade, and its' individual components (eg, hypotension, etc.) between patients with complete (disease) response at 3, 6, and/or 12 months, versus those not seeing complete response. Multivariable logistic regression was used to account for potential confounders 
in 12-month disease response. To further delineate the impacts of toxicity, we performed additional landmark analysis of time to PFS, beginning at 30 days after CART initiation. This analysis included only those patients who survived to 30 days without disease progression, stratified by toxicity grade.

In order to understand the potential contribution of specific components of CRS in association with clinical outcomes, individual components of CRS, as well as the presence of neurotoxicity, and cardiotoxicity, respectively, were considered. Blood pressure variables were included alternatively as hypotension ( $\mathrm{SBP}<80 \mathrm{~mm} \mathrm{Hg}$ ) and percent SBP decrease ranges; these were not included together in any model so as to avoid multicollinearity issues. A univariable model for overall survival was also fit with maximum CRS grade as the independent variable. We also assessed the effects of the antitoxicity interventions (tocilizumab and steroids, respectively, at grade $\leq 2$ toxicity) on the maximum grade observed. All statistical tests were two-sided and evaluated at the $\alpha=0.05$ type-I error rate, with no adjustments for multiple comparisons or evaluation of multiple cut points. All analyses were performed with SAS Software V.9.4.

\section{RESULTS}

Overall, from 102 CAR-T-treated patients, 90 were identified as treated with single-agent therapy, including 58 $(64.4 \%)$ treated with axi-cel, and $32(35.6 \%)$ with tisa-cel, respectively. The patients had a mean age of $61.0 \pm 10.9$ years and $57.8 \%$ were male; and $92.2 \%$ had an Eastern Cooperative Group performance status of $0-1$; while 94.4\% had relapsed/refractory DLBCL. Most patients were heavily pretreated, with a median number of prior treatments of $3 \pm 1.3$ (range: $0-8$ ), including $33.3 \%$ (30) who underwent prior autologous stem cell transplantation. Furthermore, $16.7 \%$ (15) of patients were treated within early trials, while $83.3 \%$ (75) received CAR-T as part of standard of care. Additional baseline and diseasespecific characteristics are described in table 1, including stratification by the degree of CRS.

\section{Incidence and severity of CRS, and other toxicities}

Following CAR-T infusion, 88.9\% (80) of patients developed CRS (figure 1), with a median time to CRS onset of 2.5 days (IQR: $1-5$ days; range: $0-9$ days). This included 98.3\% (57/58) with CRS after axi-cel, and 71.9\% (23/32) after tisa-cel infusion. Of those patients who developed CRS, $38.7 \%$ (31) saw a maximum severity of grade 2, and $16.3 \%$ (13) reached at least grade 3; with hypotension $(87.8 \%$ (79)), followed by fever $(86.7 \%$ (78)) being the most common manifestations (online supplemental figure 1; table 2); $52.2 \%$ patients experienced a minimum SBPs of $\leq 90 \mathrm{~mm} \mathrm{Hg}$. There were no specific clinical or disease-related factors associated with the development of CRS. Neurotoxicity occurred in $41(45.6 \%)$ patients, including $34(37.8 \%)$ reaching at least grade 2 (online supplemental figure 2). Furthermore, all patients with neurotoxicity experienced CRS within the same admission. In addition, 17 (18.9\%) patients developed a CVD event within days of infusion, including $11(12.2 \%)$ with arrhythmias, $2(2.2 \%)$ with myocarditis, 1 (1.1\%) heart failure, and $3(3.3 \%)$ with other CVD events (online supplemental figure 2). Among those with cardiac events, there was no difference in prevalence of concurrent or preceding high-grade $(\geq 3)$ CRS $(\mathrm{p}=0.3)$.

\section{Effect of drug product on toxicity occurrence}

The use of axi-cel was associated with a higher incidence of CRS $(98.3 \%$ vs $71.9 \%$, p $<0.01$; online supplemental table 2). The median time to CRS onset differed by product (3.0 vs 3.4 days for axi-cel and tisa-cel, respectively; $\mathrm{p}=0.45$ ), as did maximum CRS grade observed $(1.93 \pm 1.1$ vs . $1.15 \pm 1.08$ for axi-cel and tisa-cel, respectively; $\mathrm{p}=0.005$ ). Neurotoxicity was more common after axi-cel $(63.6 \%$ vs $20.3 \%, \mathrm{p}=0.001$ ). There was no difference in cardiotoxicity development, by CAR-T product. There was also no difference in PFS or mortality, by product.

\section{Toxicity development and subsequent disease response}

Over a median follow-up of 16.5 months (range: $1-54$ months), the median PFS was 6.0 months (95\% CI: 3.3 to 8.8 months), and the 25 th percentile for overall survival was 9.6 months (95\% CI: 5.1 to 14.9 months). Fifty-four (60.0\%) patients experienced disease progression or death at 12 months (online supplemental table 3). Complete remissions was attained in $42(4672 \%)$ patients at 3 months postinfusion, while nine $(10.0 \%)$ had a partial remission, and $38(42.2 \%)$ had progressive (unresponsive) disease; one (1.1\%) had stable disease. At 12 months, complete remission remained in $30(33.3 \%)$, with no partial remissions; and $54(60.0 \%)$ had progressive disease. Overall survival among all patients was $90.0 \%$ at 3 months, $80.0 \%$ at 6 months, and $72.2 \%$ at 12 months, with corresponding PFS of $57.8 \%$ at 3 months, $47.8 \%$ at 6 months, and $40.0 \%$ at 12 months. Response rates were similar to the ZUMA- ${ }^{1}$ and JULIET ${ }^{2}$ trials.

When stratified by CRS-status, maintenance of PFS at 3 months was observed among $70.0 \%$ of those with grades $0-1$ CRS, and $55.0 \%$ with grade $3-4$, and $80.0 \%$ in those with grade 2 CRS. This pattern remained through 12 months, with $36.0 \%$ maintaining PFS with grade $0-1,33.0 \%$ with grade $3-4$, and $54.0 \%$ with grade 2, respectively (figure 2). Similarly, complete disease response at 1 year was highest among those with moderate (grade 2) CRS (figure 3). These relationships remained even after accounting for other factors linked with disease and therapeutic outcomes (HR: $0.52 ; 95 \%$ CI: 0.19 to $0.88 ; \mathrm{p}=0.03$ in landmark analysis; table 3, online supplemental figure 3 and online supplemental table 4). Furthermore, similar differences in overall survival by CRS status were also observed, with moderate CRS seeing improved survival (HR: 0.43 ; $95 \%$ CI: 0.20 to 0.94 ; $\mathrm{p}=0.03$; online supplemental figure 4 and online supplemental table 4 ). 
Open access

Table 1 Baseline characteristics

\begin{tabular}{|c|c|c|c|}
\hline Variable & $\begin{array}{l}\text { Total } \\
(n=90)\end{array}$ & CRSgrade0-1†(n=46) & CRS grade $2-5 \dagger(n=44)$ \\
\hline Age, mean (SD) & $61.0(10.9)$ & $59.6(12.7)$ & $62.3(8.5)$ \\
\hline \multicolumn{4}{|l|}{ Sex, n (\%) } \\
\hline Male & $52(57.8 \%)$ & $27(58.7 \%)$ & $25(56.8 \%)$ \\
\hline Female & $38(42.2 \%)$ & $19(41.3 \%)$ & $19(43.2 \%)$ \\
\hline \multicolumn{4}{|l|}{ Race, n (\%) } \\
\hline Othert & $5(5.6 \%)$ & $4(8.7 \%)$ & $1(2.3 \%)$ \\
\hline BMI, mean (SD) & $29.0(6.5)$ & $28.0(6.2)$ & $30.0(6.6)$ \\
\hline \multicolumn{4}{|l|}{ BMI, n (\%) } \\
\hline$<25$ & $25(27.8 \%)$ & $15(32.6 \%)$ & $10(22.7 \%)$ \\
\hline $25-29.9$ & $35(38.9 \%)$ & $20(43.5 \%)$ & $15(34.1 \%)$ \\
\hline MI & $1(1.1 \%)$ & $0(0 \%)$ & $1(2.3 \%)$ \\
\hline CAD & $7(7.8 \%)$ & $4(8.7 \%)$ & $3(6.8 \%)$ \\
\hline CKD (stage IV or V) & $4(4.4 \%)$ & $3(6.5 \%)$ & $1(2.3 \%)$ \\
\hline $\mathrm{CHF}$ & $8(8.9 \%)$ & $4(8.7 \%)$ & $4(9.1 \%)$ \\
\hline $\mathrm{AF}$ & $10(11.1 \%)$ & $5(10.9 \%)$ & $5(11.4 \%)$ \\
\hline \multicolumn{4}{|l|}{ Smoking status, n, (\%) } \\
\hline Never & $43(47.8 \%)$ & $27(58.7 \%)$ & $16(36.4 \%)$ \\
\hline Previous & $38(42.2 \%)$ & $15(32.6 \%)$ & $23(52.3 \%)$ \\
\hline Current & $9(10.0 \%)$ & $4(8.7 \%)$ & $5(11.4 \%)$ \\
\hline Primary refractory & $28(31.1 \%)$ & $15(32.6 \%)$ & $13(29.5 \%)$ \\
\hline \multicolumn{4}{|l|}{ Ann Arbor stage, n (\%) } \\
\hline 0 & $0(0 \%)$ & $0(0 \%)$ & $0(0 \%)$ \\
\hline 1 & $4(4.4 \%)$ & $3(6.5 \%)$ & $1(2.3 \%)$ \\
\hline 2 & $9(10.0 \%)$ & $2(4.3 \%)$ & $7(15.9 \%)$ \\
\hline 3 & $23(25.6 \%)$ & $14(30.4 \%)$ & $9(20.5 \%)$ \\
\hline 4 & $40(44.4 \%)$ & $21(45.7 \%)$ & $19(43.2 \%)$ \\
\hline Unknown & $14(15.6 \%)$ & $6(13.0 \%)$ & $8(18.2 \%)$ \\
\hline \multicolumn{4}{|c|}{ International Prognostic Index (IPI) score, risk stratification, n (\%) } \\
\hline $0-1$ & $9(10.0 \%)$ & $6(13.0 \%)$ & $3(6.8 \%)$ \\
\hline 2 & $12(13.3 \%)$ & $7(15.2 \%)$ & $5(11.4 \%)$ \\
\hline 3 & $9(10.0 \%)$ & $4(8.7 \%)$ & $5(11.4 \%)$ \\
\hline $4-5$ & $9(10.0 \%)$ & $6(13.0 \%)$ & $3(6.8 \%)$ \\
\hline Unknown & $51(56.7 \%)$ & $23(50.0 \%)$ & $28(63.6 \%)$ \\
\hline Bulky disease & $51(56.7 \%)$ & $27(58.7 \%)$ & $24(54.5 \%)$ \\
\hline \multicolumn{4}{|c|}{ Genetic mutations, stratification, n (\%) } \\
\hline $\begin{array}{l}\text { Double hit: } \\
\text { Del-Myc and (BCL2 or BCL6) }\end{array}$ & $24(26.7 \%)$ & $10(21.7 \%)$ & $14(31.8 \%)$ \\
\hline
\end{tabular}


Table 1 Continued

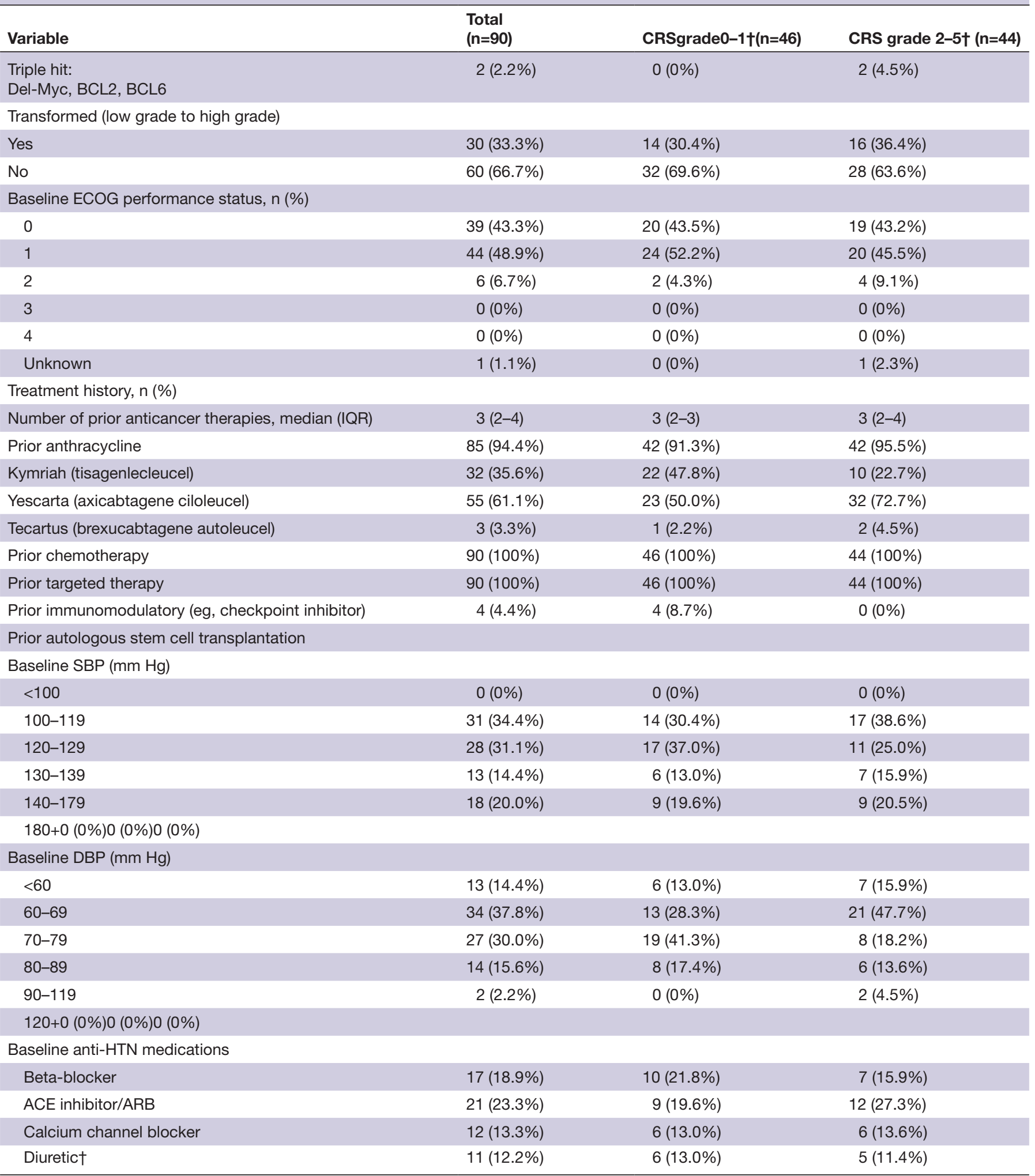

*Black, Hispanic, Asian, multiracial, and unknown race.

†Includes loop, thiazide, and potassium-sparing diuretics.

$\mathrm{ACE}$, angiotensin converting enzyme inhibitor; AF, atrial fibrillation; BCL, B-cell lymphoma; BMI, body-mass-index; CAD, coronary artery disease;

CAR-T, chimeric antigen receptor T-cell; CHF, congestive heart failure; CKD, chronic kidney disease; CVD, cardiovascular disease; DBP, diastolic

blood pressure; DLBCL, diffuse large B-cell lymphoma; DM, diabetes mellitus; ECOG, Eastern Co-operative Oncology Group; FL, follicular

lymphoma; HTN, hypertension; MCL, mantle cell lymphoma; MI, myocardial infarction; Myc, myelocytomatosis viral oncogene homolog; SBP, systolic blood pressure.; 
A

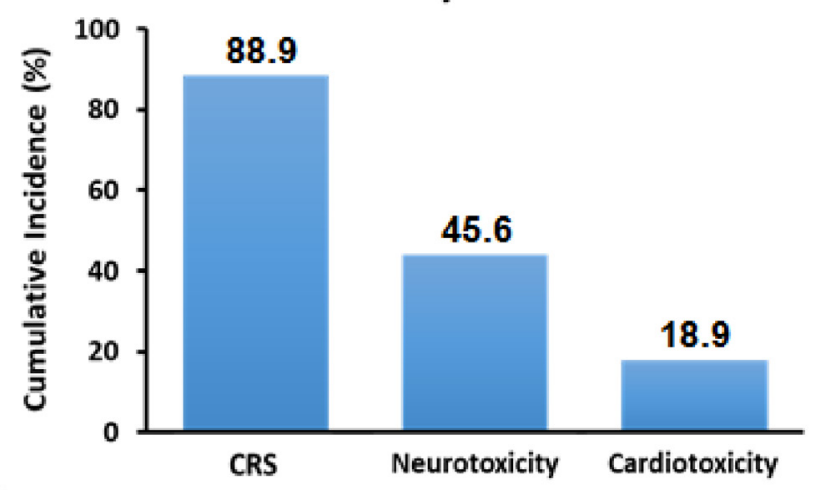

B

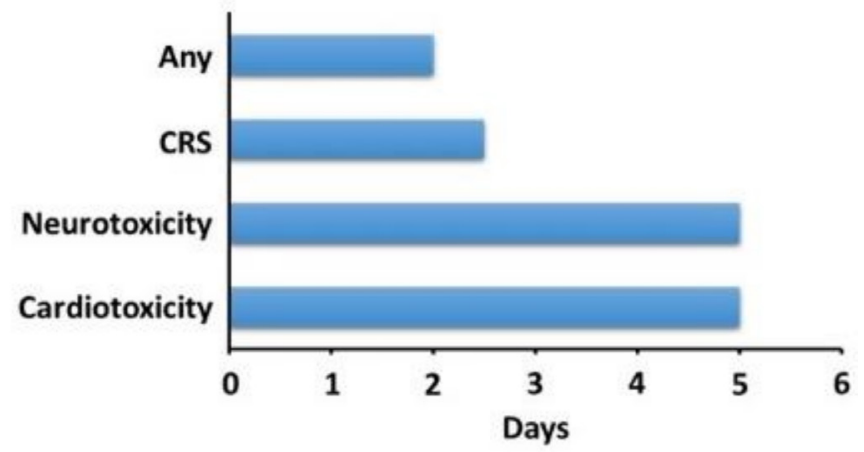

Figure 1 Cumulative incidence of early toxicities (A), and median time to toxicity development (B), after CAR-T cell infusion. CAR-T, chimeric antigen receptor T-cell; CRS, cytokine release syndrome

In univariable analysis of CRS components, hypoxia (HR: $1.88 ; 95 \%$ CI: 1.02 to $3.47 ; p=0.044$ ), coagulopathy (HR: $11.62 ; 95 \%$ CI: 4.26 to $31.67 ; \mathrm{p}<0.01$ ), organ toxicity (HR: $3.99 ; 95 \%$ CI: 1.92 to 8.33 ; $\mathrm{p}<0.01$ ), and profound hypotension (minimum SBP $<80 \mathrm{~mm} \mathrm{Hg}$; HR: 2.07; $95 \%$ CI: 1.11 to 3.87 ; $\mathrm{p}=0.02$ ) were linked with worse PFS (online supplemental table 3). Yet, in a multivariable model, only coagulopathy remained associated with worse PFS (HR: 4.72; 95\% CI: 1.22 to 18.23 ; $\mathrm{p}=0.024$; table 3 ). On stratification by temperature, there were no differences in outcomes. However, on stratification by the degree of SBP reduction, those with a minimum SBP of $80-104 \mathrm{~mm} \mathrm{Hg}$ saw PFS at 3 and 12 months of $74.0 \%$ and $50.0 \%$, respectively, compared with $70.0 \%$ at 3 months and $11.0 \%$ at 12 months in those with minimum SBPs of $\geq 105$ or the $50.0 \%$ at 3 months and $18.0 \%$ at 12 months in those with SBPs $<80 \mathrm{~mm} \mathrm{Hg}$; online supplemental figure 5 . Furthermore, by the degree of SBP change postinfusion ( $\leq 80,81-104$, or $\geq 105 \mathrm{~mm} \mathrm{Hg}$ SBP after CAR-T treatment blood pressure, and absence of active infection), SBP of $\leq 80$ or $\geq 105 \mathrm{~mm} \mathrm{Hg}$ was associated with higher PFS, which remained in multivariable analysis (HR: 2.25; 95\% CI: 1.29 to $3.91 ; \mathrm{p}<0.01$; online supplemental table 5). Relative percent fall $(\leq 10 \%,>10 \%-40 \%$, or $>40 \%$ decline in SBP from pretreatment blood pressure, and absence of active infection) in SBP of $\leq 10 \%$ or $>40 \%$ was associated
Table 2 CRS, neurotoxicity, and cardiotoxicity characteristics after CAR-T cell infusion

\begin{tabular}{ll}
\hline Variable & Summary \\
\hline Follow-up time (months), median (IQR) & $16.5(7.0-28.8)$ \\
\hline Age at CART infusion, mean (SD) & $61.0(10.9)$ \\
Sex, female & $38(42.2 \%)$ \\
Maximum CRS grade &
\end{tabular}

\begin{tabular}{|ll|}
\hline 0 & $10(11.1 \%)$ \\
\hline 1 & $36(40.0 \%)$ \\
\hline 3 & $31(34.4 \%)$ \\
\hline 4 & $6(6.7 \%)$ \\
\hline 5 & $3(3.3 \%)$ \\
\hline Specific CRS parameters & $4(4.4 \%)$ \\
\hline Fever $\left(>38^{\circ} \mathrm{C}\right)$ & \\
\hline Hypoxia & $78(86.7 \%)$ \\
\hline Organ toxicity & $19(21.1 \%)$ \\
\hline Coagulopathy & $9(10.0 \%)$ \\
\hline Hypotension $(\mathrm{SBP}<105 \mathrm{~mm} \mathrm{Hg})$ & $5(5.6 \%)$ \\
\hline Lowest SBP, mean $(\mathrm{SD})$ & $79(87.8 \%)$ \\
\hline Lowest SBP range & $90.6(13.5)$ \\
\hline
\end{tabular}

\begin{tabular}{|l|l|}
\hline$<80$ & $16(17.8 \%)$ \\
\hline $80-104$ & $63(70.0 \%)$ \\
\hline$\geq 105$ & $11(12.2 \%)$ \\
\hline$\%$ Decrease in SBP*, mean (SD) & $28.0(12.0)$ \\
\hline$\%$ Decrease in SBP & \\
\hline$\leq 10 \%$ & $5(5.6 \%)$ \\
\hline$>10 \%-40 \%$ & $71(78.9 \%)$ \\
\hline$>40 \%$ & $14(15.6 \%)$ \\
\hline Neurotoxicity & $41(45.6 \%)$ \\
\hline Neurotoxicity grade, $\mathrm{n}(\%)$ & \\
\hline
\end{tabular}

\begin{tabular}{|ll|}
\hline 0 & $49(54.4 \%)$ \\
\hline 1 & $7(7.8 \%)$ \\
\hline 2 & $12(13.3 \%)$ \\
\hline 3 & $16(17.8 \%)$ \\
\hline 4 & $5(5.6 \%)$ \\
\hline 5 & $1(1.1 \%)$ \\
\hline Steroid use & $39(43.3 \%)$ \\
\hline Tocilizumab use & $36(40.0 \%)$ \\
\hline CVD event ${ }^{*}$ & $17(18.9 \%)$ \\
\hline Clinical laboratory markers & \\
\hline CRP, median (IQR) & $21.6(8.0-67.7)$ \\
\hline Ferritin, median (IQR) & $530.0(233.5-1160.5)$ \\
\hline LDH & $224.0(182.0-273.0)$ \\
\hline PTT & $31.2(27.8-34.9)$ \\
\hline D-dimer & $0.6(0.4-2.5)$ \\
\hline Alkaline phosphatase & $77.0(64.0-101.0)$ \\
\hline
\end{tabular}

${ }^{*}$ Reflects maximum fall in systolic blood pressure from preadmission outpatient reading.

tIncludes myocarditis, myocardial infarction, congestive heart failure (CHF) symptomatic arrhythmia (eg, atrial fibrillation, ventricular tachycardia), and cardiac or sudden death $(n=90)$.

CAR-T, chimeric antigen receptor T-cell; CKD, chronic kidney disease; CRP, c-reactive protein; CRS, cytokine release syndrome; CVD, cardiovascular disease; DM, diabetes mellitus; LDH, lactate dehydrogenase; PTT, prothrombin time; SBP, systolic blood pressure.; 
A

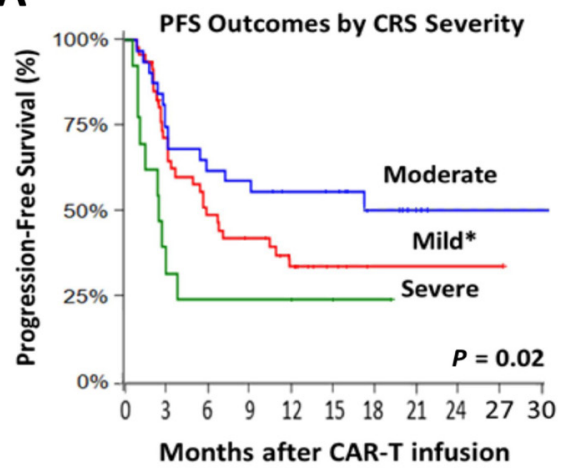

B

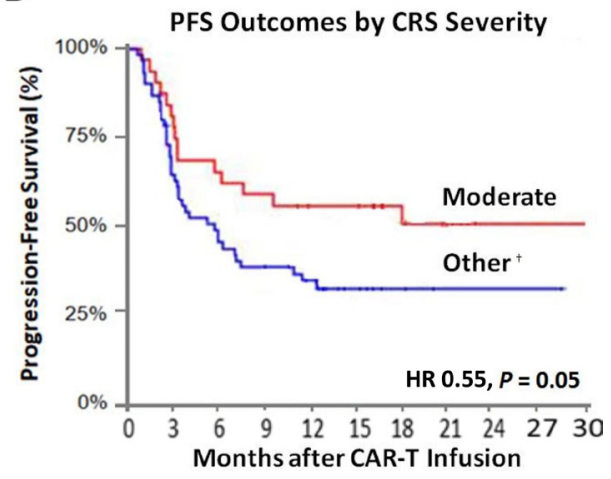

Figure 2 Kaplan-Meir curves with 30-day landmark analysis for progression-free survival across all CRS grades (mild, moderate, severe; A), and the presence or absence of moderate toxicity (B). * Reflects grades $0-1$. $†$ All grades except grade 2. CRS, cytokine release syndrome.

with higher PFS on univariate analysis, but not in multivariable analysis. There was no association between the development of other cardiovascular or neurologic events and PFS.

\section{Laboratory markers and disease response}

Where measured, the presence of CRP $(\geq 50 \mathrm{mg} / \mathrm{L})$, ferritin $(\geq 500 \mathrm{ng} / \mathrm{mL})$, and $\mathrm{LDH}(>280$ units/L) elevation, respectively, was associated with lower rates of PFS

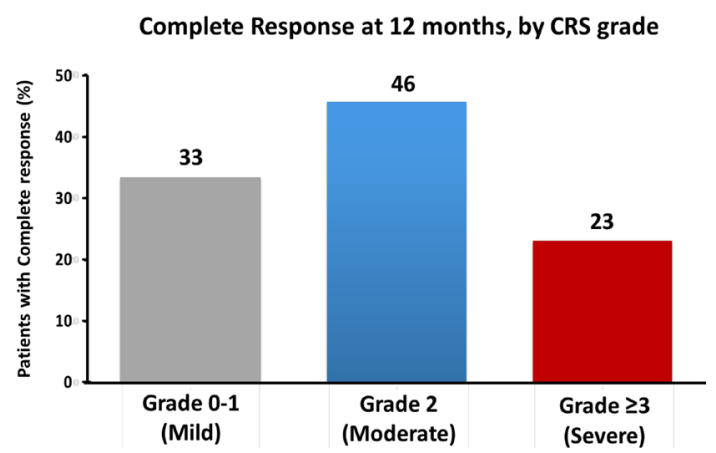

Figure 3 Maintenance of complete clinical disease response (reflected by absence of progression or malignancy related death) by 1 year after CAR-T cell infusion, according to CRS grade (severity). Responses were confirmed and assessed according to the 2014 Lugano classification for non-Hodgkin's lymphoma. CAR-T, chimeric antigen receptor T-cell; CRS, cytokine release syndrome.
Table 3A Multivariable predictors of progressionfree survival after CAR-T cell infusion, following 30-day landmark; by the Lee criteria*

\begin{tabular}{llll}
\hline Variable & HR & $\begin{array}{l}\text { 95\% Cl (lower- } \\
\text { upper) }\end{array}$ & P value \\
\hline $\begin{array}{l}\text { CRS grade 2 vs not } \\
\text { grade 2 }\end{array}$ & 0.52 & 0.28 to 0.95 & $\mathbf{0 . 0 3 3}$ \\
$\begin{array}{l}\text { Primary refractory } \\
\text { disease at CAR-T } \\
\text { initiation }\end{array}$ & 1.41 & 0.79 to 2.52 & 0.234 \\
\hline
\end{tabular}

${ }^{*} \mathrm{n}=88$.

CAR-T, chimeric antigen receptor T-cell; CRS, cytokine release syndrome.

(online supplemental figure 5). However, following multivariable adjustment, outside of ferritin, no associations with outcomes were observed (online supplemental table 4). Similarly, there was no association between prothrombin time, d-dimer, or alkaline phosphatase and disease outcomes after CAR-T infusion. Furthermore, baseline CRP was known in the majority $(72.2 \%)$ of patients, with an average delta $(\Delta)$ peak increase in CRP of $24.0 \mathrm{mg} / \mathrm{L}$, at a median of 3 days post-CART infusion. Among those with available measures, there was a correlation between $\triangle$ CRP and maximum CRS grade $\left(\mathrm{r}^{2}=0.28\right.$, $\mathrm{p}=0.02)$.

\section{Grading system selection and PFS outcomes}

The effect of CRS grading system on PFS was assessed based on the three primary CRS grading criteria: Penn, Transplantation and Cellular Therapy(TCT), and Lee, respectively. The variance across the three scoring scales was small. The proportion of patients with grade 2 CRS

Table 3B Multivariable predictors of progression-free survival after CAR-T cell infusion, by toxicity type and component*

\begin{tabular}{llll}
\hline Variable & HR & $\mathbf{9 5 \%} \mathbf{C l}$ (lower-upper) & P value \\
\hline Fever $^{\star}\left(>38^{\circ} \mathrm{C}\right)$ & 0.36 & 0.27 to 1.43 & 0.269 \\
\hline Hypoxia† & 1.45 & 0.63 to 3.32 & 0.376 \\
\hline Organ toxicity $\ddagger$ & 2.26 & 0.66 to 7.70 & 0.190 \\
Coagulopathy & 4.72 & 1.22 to 18.23 & $\mathbf{0 . 0 2 4}$ \\
Hypotension§ & 1.33 & 0.53 to 3.35 & 0.532 \\
Neurotoxicity & 0.62 & 0.30 to 1.25 & 0.183 \\
\hline
\end{tabular}

The $\mathrm{P}$ values that are bolded are the ones that are statistically significant.

${ }^{*}$ Maximum temperature $>38^{\circ} \mathrm{C}$.

†Requiring supplemental oxygen (nasal cannula oxygen, high flow oxygen, continuous positive airway pressure or bilevel positive airway pressure, or intubation).

¥Other than cardiac or neurologic organ toxicity, $n=90$.

§Minimum systolic blood pressure of $<80 \mathrm{~mm} \mathrm{Hg}$.

CAR-T, chimeric antigen receptor T-cell; CKD, chronic kidney disease; CRS, cytokine release syndrome; DM, diabetes mellitus; SBP, systolic blood pressure. 
Table 3C Multivariable predictors of complete response at 12 months after CAR-T cell infusion*

\begin{tabular}{llll}
\hline Variable & HR & $\begin{array}{l}\text { 95\% Cl (lower- } \\
\text { upper) }\end{array}$ & P value \\
\hline $\begin{array}{l}\text { CRS grade 2 vs not } \\
\text { grade 2 }\end{array}$ & 2.47 & 1.00 to 6.08 & $\mathbf{0 . 0 4 8}$ \\
$\begin{array}{l}\text { Primary refractory } \\
\text { disease at CAR-T } \\
\text { initiation }\end{array}$ & 0.80 & 0.31 to 2.09 & 0.657 \\
\hline
\end{tabular}

*One subject had 16 month follow-up data, rather than at 12 months, $n=90$.

CAR-T, chimeric antigen receptor T-cell; CRS, cytokine release syndrome.

was $34.4 \%$ by Penn criteria, $34.4 \%$ by Lee criteria, and $38.9 \%$ by TCT criteria. There was no difference in the relationship between grading system and PFS $(p=0.9995$, online supplemental figure 6 ). Furthermore, on multivariate analysis, a similar degree of significance was maintened between the relationship of $\mathrm{b}$ moderate CRS and PFS outcomes, by Penn, TCT, and Lee criteria $(p=0.067$, 0.067 , and 0.033 , respectively; online supplemental table 6 ); with axicel showing stronger associations (HR: 0.40; 95\% CI: 0.18 to $0.87 ; \mathrm{p}=0.02$; online supplemental tables 7 and 8).

\section{Impact of toxicity reducing interventions}

Tocilizumab, an interleukin-6 inhibitor, was used in 36 $(40.0 \%)$ patients due to worsened CRS, with a mean CRS grade of $1.87 \pm 0.63$ (median grade of 2) at the time of initiation. There was no difference in mortality $(\mathrm{p}=0.91)$, CRS worsening, cardiotoxic or neurotoxic events, by tocilizumab-status $(\mathrm{p}=0.99)$. Furthermore, steroids were used in $39(43.3 \%)$ patients due to neurotoxicity (and three patients received steroids for CRS), with a mean toxicity grade at the time of initiation of $2.17 \pm 0.71$ (median grade 2). Similarly, there was no difference in mortality $(\mathrm{p}=0.538)$, including in those with concurrent CRS $(p=0.573)$; or the likelihood of neurotoxicity worsening $(p=0.594)$, or cardiotoxic events $(p=0.99)$. Furthermore, there was no difference in PFS or disease response based on the presence or absence of tocilizumab or steroid use, respectively (online supplemental table 4).

\section{DISCUSSION}

In this evaluation of the prognostic implications of toxicity after CAR-T infusion, more than $89 \%$ of patients developed CRS or other toxicities. Those patients who developed moderate CRS (grade 2) saw marked improvement in the outcomes of PFS, objective disease response, and overall survival compared with those with no/minimal or severe CRS. This pattern remained, even after accounting for the timing of CRS development, and pretreatment disease severity. Moreover, considering the specific components of CRS, the presence of post-therapy fever and hypotension strongly associated with higher therapeutic response and the avoidance of disease-progression. Furthermore, the development of a post-treatment intermediate hemodynamic response to therapy, reflected by a 10\%-40\% fall in SBP after infusion, was associated with a nearly threefold increase in the likelihood of longer term PFS. These observations are of particular importance, given the rapidly increasing prevalence of CAR-T use, and the lack of available early markers to guide the determination of likelihood of post-treatment long-term disease response.

The observation of improved anticancer efficacy after early treatment-related toxicity adds to a growing body of evidence linking cancer immunomodulatory therapy related adverse events with clinical disease outcomes. ${ }^{14-19}$ In prior studies of relapsed lymphoma patients treated with programmed cell death ligand inhibitor-1, nivolumab, the development of adverse events within weeks of treatment initiation was associated with a nearly twofold improvement in long-term objective disease control, and PFS. ${ }^{14}$ Similarly, the occurrence of early toxicity was associated with a two to fivefold improvement in PFS among nivolumab treated non-small cell lung and melanoma patients. ${ }^{15} 16$ This pattern of association between anticancer therapeutic efficacy and adverse events has also been noted following other immunotherapies, including interferon, interleukin-2, and talimogene laherparepvec therapy. ${ }^{17-19}$ Knowledge of these effects has dramatically altered the understanding of cancer cellular biology, anticancer therapeutic efficacy, toxicity, and diseaseprognostication. This has generally pervaded the relative acceptance of some toxicities after the initiation of clinically efficacious therapies, particularly if linked with therapeutic effect. Yet, to the best of our knowledge, this is the first investigation linking toxicity with therapeutic efficacy after CAR-T infusion. The implications of these findings may bear weight on the understanding and interpretation of the adverse events after the initiation of CAR-T based therapies.

Knowledge of the indicators of CAR-T activity in patients with advanced malignant disease is of substantial importance. Currently, the primary available tools for monitoring the anticancer efficacy of CAR-T includes postdischarge PET disease activity at least 90 days after CAR-T infusion (in those with lymphomas). ${ }^{25}$ And although other techniques have been proposed, including cytokine profiling, enumeration of tumor-specific T-cells in peripheral blood, and invasive tumor biopsies, their use has been confounded by CRS syndrome and the need for invasive monitoring. ${ }^{26}$ Identification of more practical early tools, including the development and degree of toxicity itself, represent a step forward for clinicians tasked with decisions regarding subsequent timely treatment strategies. Given the high correlation between moderate CRS, a fall in SBP of $10 \%-40 \%$, and subsequent PFS, it is plausible that this prognostic variable could be used to identify patients where early PET imaging (eg, 30 days) may be beneficial. Specifically, this may allow for the initiation 
of early alternative interventions targeted at enhanced augmentation of CAR-T activity, and the opportunity for improved longer term clinical responses. Nevertheless, with the high prevalence of CRS, initially described in the early phase trials, identification and potential interpretation of these common events may prove beneficial to the rapid assessment of therapeutic efficacy following the initiation of these therapies.

Despite a general awareness of the prevalent nature of toxicity after CAR-T infusion, variation in the reported incidence of CRS has been previously observed. In the pivotal ZUMA-1 trial, CRS was observed among 93\% of patients after axi-cel therapy (using the National Institutes of Health consensus criteria), with $59 \%$ developing significant hypotension. ${ }^{1}$ In the JULIET trial, $58 \%$ of patients developed CRS using the Penn toxicity criteria, with $22 \%$ being grade 3 or higher, and $21 \%$ of patients developed neurotoxicity, with $12 \%$ being grade $\geq 3$. ${ }^{3}$ This effect has also been seen with other emerging forms of CAR-T-based therapies. ${ }^{6} 7$ Moreover, in these studies, the occurrence of more significant ( $\geq$ grade 2 ) events has been reported to be over $55 \%$. However, due to the heterogeneity in CRS grading, and the innate differences between CAR-T constructs, adequate comparisons across studies have been limited. Given the high prevalence and the potential impact, these events may portend for disease response, consistent application of uniform criteria, including recent proposals by the American Society for Transplantation and Cellular Therapy to standardize CRS assessments may prove vital to the understanding and evaluation of CAR-T-related toxicity. ${ }^{27}$ The observation of stronger associations between toxicity and outcomes with axi-cel, accounting for some biologic T-cell activation difference, may also warrant prospective investigation to further delineate the potential impacts of product selection.

Although the exact reasons behind the association of early toxicity with CAR-T outcomes is unknown, several plausible mechanisms may underlie these findings. The level of CRS severity has been previously linked to higher pretreatment disease burdens. ${ }^{5}$ Accordingly, it is plausible that the occurrence of these phenomena may simply be due to the cellular expansion and activation of immune cell subsets with inflammation, and thus act as an early surrogate for longer term disease response. However, this consideration is limited by the lack of association between high grades $(\geq 3)$ of CRS and reduction in the rate of continued disease remission. Similarly, a link between the development of other forms of toxicity, including cardiovascular and neurotoxicity was not seen. The occurrence of more moderate degrees of CRS may reflect a stable, but optimal level of immune response to CAR-T therapy, as observed with other immunotherapies. ${ }^{14-19}$ Given the early report of reasonable disease response with other investigational CAR-T therapies linked with less prevalent manifestations of CRS, it may be likely that the degree of clinical sequelae with established therapies may more clearly reflect the nature of the relationship of toxicity to disease response. Within the current analysis, hypotension was observed to critically predict future disease response. Vascular leak has been previously proposed to contribute to this clinical phenomena. ${ }^{26} \mathrm{CAR}-\mathrm{T}$ treatment may induce change in T-cell morphology, prompting abrupt cellular volume expansion and extracellular fluid loss and subsequent intravascular depletion. Yet, with the commonly used CD19-specific CAR-Ts, this relationship has not been observed. In conjunction with prospective evaluations of the prognostic role of early toxicity (including cardiac), additional mechanistic studies are needed.

\section{Study limitations}

Several limitations should be acknowledged. Given the retrospective nature of the study, available follow-up within the cohort was not uniform. Also, we could not exclude information bias. Uniform cardiac, neurologic, and correlative laboratory testing protocols were not in place near the initiation of programmatic CAR-T-based treatments, as many patients were in early phase clinical trials. Similarly, timely tocilizumab and steroid application was at the discretion of the treating clinicians and thus varied across time. CART management strategies shifted over time. Notably, the proportion of patients receiving tisa-cel increased after FDA approval in 2018. Furthermore, tocilizumab was utilized more frequently in practice than was initially outlined in the prior JULIET and ZUMA-1 trials. ${ }^{2}$ Despite these factors, the data represent real-world practice, and are thus generalizable to this population. Although we did not observed mitigation in maximum toxicity grade by intervention antitoxicity therapies, it is not known if treatment in combination with supportive therapies (eg, fluids), may have shortened toxicity durations, ${ }^{28}$ or prevented some patients from reaching high grades or death. We did not include children. Relationships with axicel were more robust. Keppra was not commonly used for seizure prophylaxis. The population consisted primarily of DLBCL patients, and it is unclear if the same relationships would be observed among non-lymphoma patients. The current study focused on relapsed and refractory lymphoma patients treated with axi-cel and tisa-cel, respectively, and did not include data from more recently available CAR-T products or other disease populations. Pooled product data were employed for most analyses, as sample size limited further product subgroup analyses. Yet, there was no difference in PFS by CAR-T product used. However, given the desire to best reflect commonly used CAR-Ts in contemporary clinical practice, we focused on these highprofile and more available therapies. Additionally, given that the risk of non-CRS toxicities (namely cardiotoxicity) was not known during the early experience with CAR-T therapy, several adverse events may have gone uncaptured despite extensive search.

\section{CONCLUSION}

CAR-T therapy is associated with an elevated risk of early toxicity. However, the occurrence of a moderate degree 
of this toxicity appears to associate with long-term disease response and PFS. This suggest that the recognition of early clinical events may enhance the early interpretation of the therapeutic effects of CAR-T-based therapy, and potentially provide the opportunity for the earlier identification of patients likely to need additional diseasespecific interventions. Given the anticipated increase in CAR-T use, additional studies of the role of early toxicity as a potential prognostic biomarker, in the setting of severity limiting interventions are needed.

\section{Author affiliations}

${ }^{1}$ Bone Marrow Transplantation and Cellular Therapies Program, Division of Hematology, The Ohio State University James Cancer Hospital, Columbus, Ohio, USA ${ }^{2}$ Internal Medicine, The Ohio State University Wexner Medical Center, Columbus, Ohio, USA

${ }^{3}$ Cardio-Oncology Program, Division of Cardiology, The Ohio State University Wexner Medical Center, Columbus, Ohio, USA

${ }^{4}$ Center for Biostatistics, Department of Biomedical Informatics, The Ohio State University Wexner Medical Center, Columbus, Ohio, USA

${ }^{5}$ Cardiology, University Hospitals Harrington Heart \& Vascular Institute, Cleveland, Ohio, USA

${ }^{6}$ Division of Hematology, The Ohio State University James Cancer Hospital, Columbus, Ohio, USA

${ }^{7}$ National Marrow Donor Program, Minneapolis, Minnesota, USA

${ }^{8}$ Division of Cancer Control and Prevention, The Ohio State University James Cancer Hospital, Columbus, Ohio, USA

\section{Acknowledgements The authors acknowledge and thank the patients and their} families treated at the Ohio State University Comprehensive Cancer Center.

Contributors Concept and design: JEB, ZB, and DA. Acquisition, analysis, or interpretation of data: JEB, ZB, AK, MB, and DA. Drafting of the manuscript: JEB, $\mathrm{ZB}, \mathrm{KP}$, and $\mathrm{DA}$. Critical revision of the manuscript for important intellectual content: All authors. Statistical analysis: KP, AG, VYO, and BB. Administrative, technical, or material support: JEB, SJ, and DA. Supervision: JEB and DA.

Funding This work was supported, in part, by National Cancer Institutes (NCI) grant P30 CA016058, and by National Institutes of Health (NIH) grants KL2-TR002734 (Dr Brammer), R01HL127442-01A1 (Dr Gumina), P30CA016058 (Dr Addison), K23HL155890 (Dr. Addison) and K12CA133250 (Dr Addison), and by American Heart Association-Robert Wood Johnson Foundation (Harold Amos) grant (Dr Addison).

Disclaimer The manuscript's content is solely the responsibility of the authors and does not necessarily represent the official views of the National Institutes of Health.

Competing interests None declared.

Patient consent for publication Not required.

Ethics approval The study was approved under the Ohio State University Comprehensive Cancer Center Institutional Review Board.

Provenance and peer review Not commissioned; externally peer reviewed.

Data availability statement Data are available upon reasonable request. All data relevant to the study are included in the article or uploaded as supplementary information. All data relevant to the study are included in the article or uploaded as supplementary information. Furthermore, any additional data are available upon reasonable request.

Supplemental material This content has been supplied by the author(s). It has not been vetted by BMJ Publishing Group Limited (BMJ) and may not have been peer-reviewed. Any opinions or recommendations discussed are solely those of the author(s) and are not endorsed by BMJ. BMJ disclaims all liability and responsibility arising from any reliance placed on the content. Where the content includes any translated material, BMJ does not warrant the accuracy and reliability of the translations (including but not limited to local regulations, clinical guidelines, terminology, drug names and drug dosages), and is not responsible for any error and/or omissions arising from translation and adaptation or otherwise.

Open access This is an open access article distributed in accordance with the Creative Commons Attribution Non Commercial (CC BY-NC 4.0) license, which permits others to distribute, remix, adapt, build upon this work non-commercially, and license their derivative works on different terms, provided the original work is properly cited, appropriate credit is given, any changes made indicated, and the use is non-commercial. See http://creativecommons.org/licenses/by-nc/4.0/.

ORCID iD

Zachary Braunstein http://orcid.org/0000-0003-2517-9943

\section{REFERENCES}

1 Neelapu SS, Locke FL, Bartlett NL, et al. Axicabtagene ciloleucel CAR T-cell therapy in refractory large B-cell lymphoma. N Engl J Med 2017;377:2531-44.

2 Schuster SJ, Bishop MR, Tam CS, et al. Tisagenlecleucel in adult relapsed or refractory diffuse large B-cell lymphoma. N Engl J Med 2019;380:45-56.

3 Lee DW, Kochenderfer JN, Stetler-Stevenson M, et al. T cells expressing CD19 chimeric antigen receptors for acute lymphoblastic leukaemia in children and young adults: a phase 1 dose-escalation trial. Lancet 2015;385:517-28.

4 Maude SL, Frey N, Shaw PA, et al. Chimeric antigen receptor T cells for sustained remissions in leukemia. $N$ Engl J Med 2014;371:1507-17.

5 Locke FL, Ghobadi A, Jacobson CA, et al. Long-Term safety and activity of axicabtagene ciloleucel in refractory large B-cell lymphoma (ZUMA-1): a single-arm, multicentre, phase 1-2 trial. Lancet Oncol 2019;20:31-42.

6 Raje N, Berdeja J, Lin Y, et al. Anti-BCMA CAR T-cell therapy bb2121 in relapsed or refractory multiple myeloma. $N$ Engl J Med 2019;380:1726-37.

7 Yan Z, Cao J, Cheng $\mathrm{H}$, et al. A combination of humanised anti-CD19 and anti-BCMA CAR T cells in patients with relapsed or refractory multiple myeloma: a single-arm, phase 2 trial. Lancet Haematol 2019;6:e521-9.

8 U.S. Food and Drug Administration. FDA approves CAR-T cell therapy to treat adults with certain types of large B-cell lymphoma. Available: https://www.fda.gov/news-events/press-announcements/ fda-approves-car-t-cell-therapy-treat-adults-certain-types-large-bcell-lymphoma [Accessed 30 Nov 2020].

9 U.S. National Library of Medicine. Available: https://clinicaltrials. gov/ct2/results?term $=\mathrm{CAR}+\mathrm{T}+\mathrm{Cell} \&$ Search $=$ Apply\&recrs=b\&recrs $=$ $a \& r e c r s=f \& r e c r s=d \& a g e \_v=\& g n d r=\& t y p e=\& r s l t=[$ Accessed $11 \mathrm{Nov}$ 2020].

10 Jacobson C, Emmert A, Rosenthal MB. Car T-cell therapy: a microcosm for the challenges ahead in Medicare. JAMA 2019;322:923-4.

11 Grigor EJM, Fergusson D, Kekre N, et al. Risks and benefits of chimeric antigen receptor T-cell (CAR-T) therapy in cancer: a systematic review and meta-analysis. Transfus Med Rev 2019;33:98-110.

12 Alvi RM, Frigault MJ, Fradley MG, et al. Cardiovascular events among adults treated with chimeric antigen receptor T-cells (CAR-T). J Am Coll Cardiol 2019;74:3099-108.

13 Guha A, Addison D, Jain P, et al. Cardiovascular events associated with chimeric antigen receptor $T$ cell therapy: cross-sectional FDA adverse events reporting system analysis. Biol Blood Marrow Transplant 2020;26:2211-6.

14 Haratani K, Hayashi H, Chiba Y, et al. Association of immune-related adverse events with nivolumab efficacy in non-small-cell lung cancer. JAMA Oncol 2018;4:374-8.

15 Eggermont AMM, Kicinski M, Blank CU, et al. Association between immune-related adverse events and recurrence-free survival among patients with stage III melanoma randomized to receive pembrolizumab or placebo: a secondary analysis of a randomized clinical trial. JAMA Oncol 2020;6:519-27.

16 Freeman-Keller M, Kim Y, Cronin H, et al. Nivolumab in resected and unresectable metastatic melanoma: characteristics of immunerelated adverse events and association with outcomes. Clin Cancer Res 2016;22:886-94.

17 Zinzani PL, Ribrag V, Moskowitz $\mathrm{CH}$, et al. Safety and tolerability of pembrolizumab in patients with relapsed/refractory primary mediastinal large B-cell lymphoma. Blood 2017;130:267-70.

18 Gogas H, loannovich J, Dafni U, et al. Prognostic significance of autoimmunity during treatment of melanoma with interferon. $N$ Engl J Med 2006;354:709-18.

19 Curti B, Daniels GA, McDermott DF, et al. Improved survival and tumor control with Interleukin-2 is associated with the development of immune-related adverse events: data from the PROCLAIM ${ }^{\text {SM }}$ registry. J Immunother Cancer 2017;5:102. 
20 Porter D, Frey N, Wood PA, et al. Grading of cytokine release syndrome associated with the CAR T cell therapy tisagenlecleucel [published correction appears in J Hematol Oncol. 2018 Jun 13;11(1):81]. J Hematol Oncol 2018;11:35.

21 Division of Cancer Treatment and Diagnosis. Common terminology criteria for adverse events (CTCAE), version 5.0. Bethesda, MD: National Cancer Institute, 2017. https://ctep.cancer.gov/ protocolDevelopment/electronic applications/ctc.htm\#ctc 50

22 Cheson BD, Fisher RI, Barrington SF, et al. Recommendations for initial evaluation, staging, and response assessment of Hodgkin and non-Hodgkin lymphoma: the Lugano classification. J Clin Oncol 2014;32:3059-67.

23 Brudno JN, Kochenderfer JN. Toxicities of chimeric antigen receptor T cells: recognition and management. Blood 2016;127:3321-30.
24 Neelapu SS, Tummala S, Kebriaei P, et al. Chimeric antigen receptor T-cell therapy - assessment and management of toxicities. Nat Rev Clin Oncol 2018;15:47-62.

25 Krebs S, Ponomarev V, Slovin S, et al. Imaging of car T-cells in cancer patients: paving the way to treatment monitoring and outcome prediction. J Nucl Med 2019;60:879-81.

$26 \mathrm{Hu}$ Y, Feng J, Shao M, et al. Profile of capillary-leak syndrome in patients received chimeric antigen receptor T cell therapy. Blood 2018;132:5204.

27 Lee DW, Santomasso BD, Locke FL, et al. ASTCT consensus grading for cytokine release syndrome and neurologic toxicity associated with immune effector cells. Biol Blood Marrow Transplant 2019;25:625-38.

28 Le RQ, Li L, Yuan W, et al. Fda approval summary: tocilizumab for treatment of chimeric antigen receptor T cell-induced severe or lifethreatening cytokine release syndrome. Oncologist 2018;23:943-7. 